\title{
Using Magnetic Flux Conservation to Determine Heliosheath Speeds
}

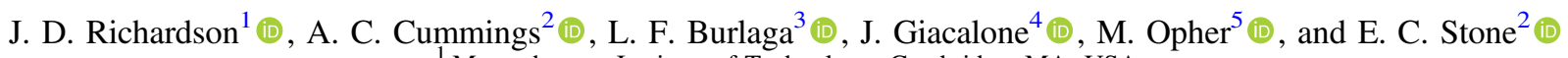 \\ ${ }^{1}$ Massachusetts Institute of Technology, Cambridge, MA, USA \\ ${ }^{2}$ California Institute of Technology, Pasadena, CA 91125 USA \\ ${ }^{3}$ NASA Goddard Space Flight Center, Greenbelt, MD, USA \\ ${ }^{4}$ The University of Arizona, Tucson, AZ, USA \\ 5 Boston University, Boston, MA, USA \\ Received 2021 July 18; revised 2021 September 14; accepted 2021 September 15; published 2021 October 4
}

\begin{abstract}
The heliosheath (HSH) radial speeds at Voyager 2 (V2) derived from the plasma instrument (PLS) and from particle instruments using the Compton-Getting (CG) effect are different. At V2 the CG speeds are more variable than the plasma speeds and decrease about 2 yr before the heliopause. We use magnetic flux conservation to differentiate between these two speed profiles at V2, comparing the magnetic flux observed at 1 au and in the HSH. For V2 the PLS speed profile is significantly more consistent with magnetic flux conservation than the CG speeds. For Voyager 1 (V1), we present new $V_{\mathrm{R}}$ derivations from the Cosmic Ray Subsystem (CRS) using the CG method that agree reasonably well with those previously obtained from the low energy charged particle (LECP) instrument. If we use the V2 PLS speed profile to calculate the magnetic flux at V1, we again find much better agreement than if we use the V1 CG speeds. These results suggest that the radial speeds derived from particle anisotropy observations in the HSH are not reliable.
\end{abstract}

Unified Astronomy Thesaurus concepts: Heliosheath (710)

\section{Introduction to the Heliosheath}

Voyagers 1 and 2 (V1 and V2) have exited the heliosphere and entered the interstellar medium. On the way out they both traversed the heliosheath (HSH), the region of shocked solar wind between the termination shock and heliopause (HP). V1 was at about $34^{\circ} \mathrm{N}$ and $\mathrm{V} 2$ was at about $30^{\circ} \mathrm{S}$ heliolatitude as they crossed the HSH. In longitude, V1 is near the nose of the heliosphere and V2 was further to the flank, about $40^{\circ}$ from the nose (Bzowski et al. 2015).

Krimigis et al. (2011) reported that V1 entered a stagnation region 8 au in front of the heliopause where the radial speed $V_{R}$ was near zero and the tangential speed $V_{\mathrm{T}}$ was small. (We use the RTN coordinate system that has $R$ radially outward from the Sun, $T$ parallel to the solar equator and positive in the direction of solar rotation, and $N$ completes the right-handed system.) Subsequent observations showed that the normal speed $V_{\mathrm{N}}$ was also small (Stone \& Cummings 2011; Decker et al. 2012). The V1 speed components were derived from the low energetic charged particle (LECP) instrument $28-53 \mathrm{keV}$ ion observations using the Compton-Getting (CG) effect (Krimigis et al. 2011; Richardson \& Decker 2014). In this Letter we present new derivations of the V1 radial speeds from the Cosmic Ray Subsystem (CRS) using $\sim 0.5-35 \mathrm{MeV} /$ nuc ion observations and the CG effect. Direct plasma measurements are not available from V1 since the plasma experiment failed in 1980.

The V2 plasma experiment did not observe a stagnation region in the $\mathrm{HSH} ; V_{\mathrm{R}}$ remained positive and $>60 \mathrm{~km} \mathrm{~s}^{-1}$ until just before the HP (Richardson et al. 2019). Average LECP $V_{R}$ speeds were similar to those reported by the plasma instrument (PLS) before 2016, but with many excursions to lower and higher $V_{\mathrm{R}}$ values (Richardson \& Decker 2014). The LECP team reported a decrease in average V2 CG-derived $V_{\mathrm{R}}$ values starting about 8 au before the HP (Krimigis et al. 2019). In this region, which is similar in width to the stagnation region at V1, the LECP CG $V_{\mathrm{R}}$ values are below those observed by PLS (Richardson et al. 2020).

Cummings et al. (2021) analyzed V2 CRS data during spacecraft rolls, which normally occur every few months. They used the observed anisotropies of $\sim 0.5-35 \mathrm{MeV} /$ nuc ions, higher energy than the $28-53 \mathrm{keV}$ ions used for the LECP analysis, to derive CG $V_{R}$ values. Despite using different energy ions, they found $V_{\mathrm{R}}$ speeds consistent with the LECP values and thus different from the PLS values. They suggest that this discrepancy between PLS and LECP speeds calls into question the accuracy of the CG calculations and casts doubt on the existence of a stagnation region at V1. An alternate explanation would be that the PLS $V_{\mathrm{R}}$ values at V2 are incorrect.

This discrepancy between the PLS and CG speeds is key to understanding the HSH; we need to determine whether the PLS or CG (CRS and LECP) $V_{\mathrm{R}}$ values are correct. These values are used to verify global MHD models and to calculate ENA fluxes for comparison with data (Fuselier et al. 2021). One way to distinguish between these two data sets is to compare the magnetic flux measured at 1 au to that obtained using the different Voyager velocity profiles. The magnetic flux is shown to be a conserved quantity by both theory (Parker 1963) and observations (Burlaga et al. 2021). We show that the V2 PLS $V_{\mathrm{R}}$ values are consistent with magnetic flux conservation and the $C G$ values are not.

\section{Data}

The PLS instrument measures ion currents directly in four Faraday cups; three point sunward and one looks at right angles to this direction (Bridge et al. 1977). When good data are available in at least three of these cups, convected isotropic proton Maxwellian distributions are fit to the data to derive the proton velocity, density, and temperature. The average $1 \sigma$ uncertainty in $V_{\mathrm{R}}$ is about $10 \%$ (Richardson \& Decker 2014). The CG speeds are calculated from observations 


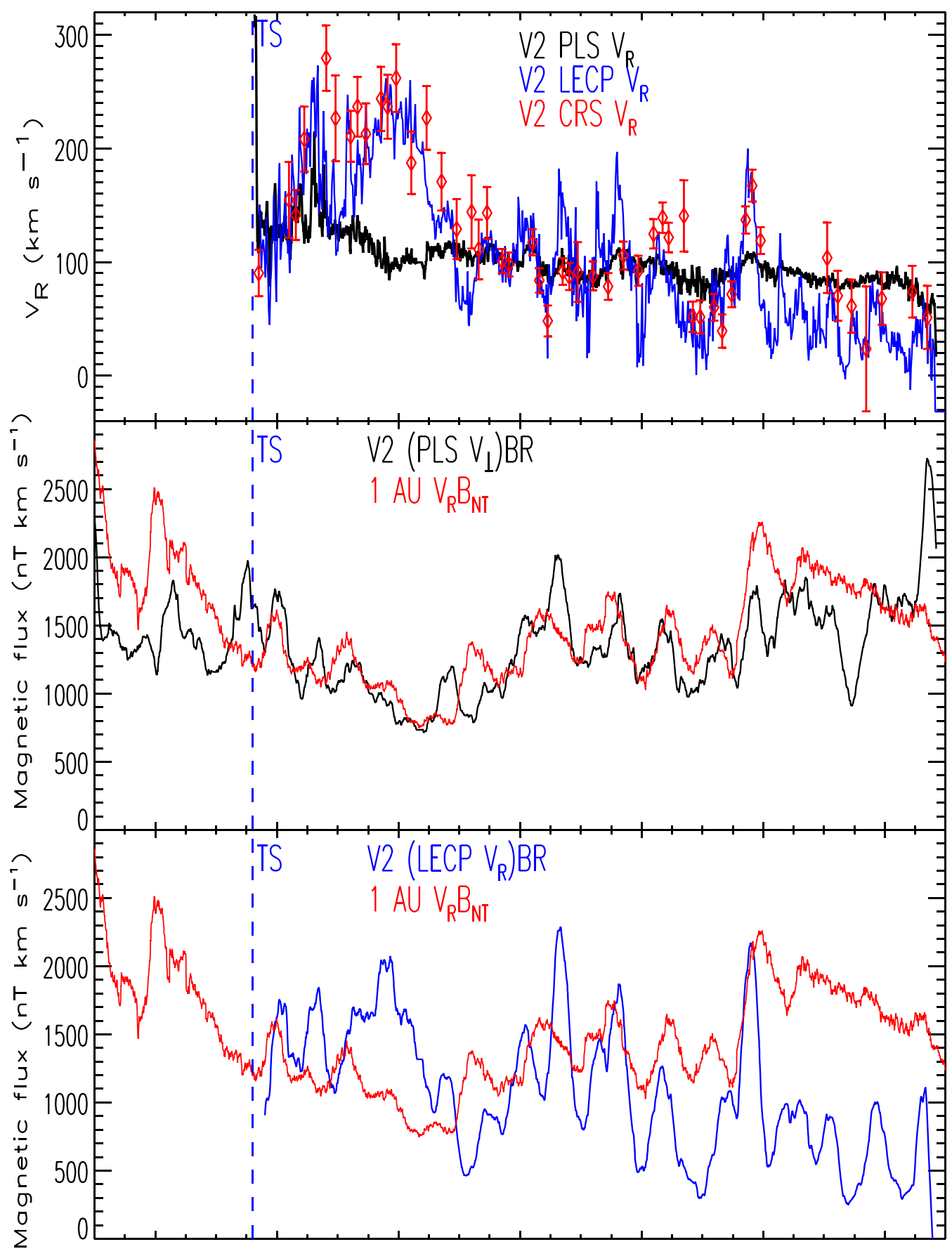

Figure 1. Top: V2 HSH radial speeds; 5 day running averages from PLS observations (black), 5 day averages from LECP CG calculations (blue), and CG calculations from CRS during rolls (red diamonds with $1 \sigma$ error bars). The LECP error bars are not shown to make the plots clear but average about $15 \mathrm{~km} \mathrm{~s}^{-1}$. Middle: 99 day running averages of the magnetic flux from the 1 au OMNI data (red) compared with 25 day running averages of the V2 magnetic flux calculated using the PLS speeds (black). The 1 au data are time-shifted forward $1 \mathrm{yr}$ to account for the propagation time from 1 au to the HSH. Bottom: 99 day running average of magnetic flux from 1 au (red) compared with 25 day running averages of the V2 magnetic flux calculated using the LECP speeds (blue). The 1 au data are time-shifted forward 1 yr to account for the propagation time from 1 au to the HSH.

of particle fluxes in different look directions, assuming the distribution is isotropic in the moving frame, and finding the speed of that frame. The LECP detector steps through eight look directions in roughly the RT plane and gets continuous anisotropy measurements. We use 5 day $V_{\mathrm{R}}$ averages from V2 (Krimigis et al. 2019) and 26 day average data from V1 (Krimigis et al. 2011). The CRS instrument (Stone et al. 1977) measures anisotropies when the spacecraft rolls that are used to derive the speed components (Cummings et al. 2021). The magnetometer provides Voyager data at $48 \mathrm{~s}$ intervals from both V1 and V2. We use daily average data Voyager magnetic fields obtained from SPDF.

\section{Magnetic Flux Conservation}

Parker (1963) demonstrates that the magnetic flux in the solar wind is a conserved quantity, with significant losses due only to reconnection. The magnetic flux is removed from the heliosphere since it is carried with the solar wind down the heliotail. Richardson et al. (2013) show that the average 


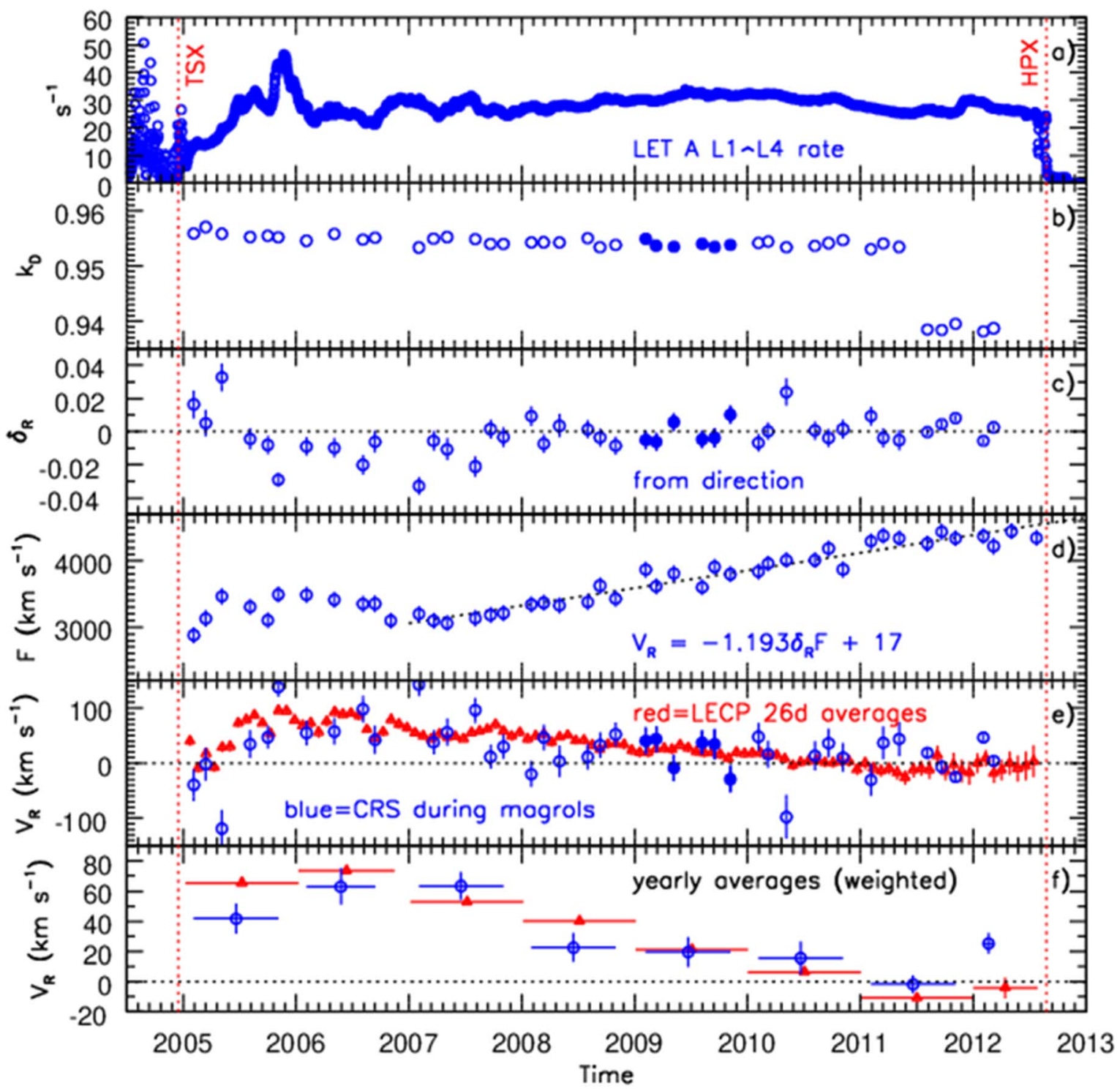

Figure 2. (a) Counting rate from the V1 CRS LET A L1 detector (with the L4 detector in anticoincidence). (b) Normalization factor for the LET D rate as described in the text. The abrupt change in 2011 is because of a command state change for LET D that made it have the same coincidence condition as LET A. The command also made LET D a continuous rate, the same as LET A, rather than a subcommutated rate. The solid circles in 2009 represent data that were used in the normalization procedure to give approximately the same $V_{\mathrm{R}}$ as that from LECP. (c) $R$ component of the anisotropy vector of $\sim 0.5-35 \mathrm{MeV}$ protons derived from fits to an assumed first-order anisotropy model of the intensity. The values are for the $\mathrm{S} / \mathrm{C}$ frame of reference and positive values denote the direction the particles are arriving from. (d) CG factor that converts between a measured anisotropy and a solar wind speed. The values are from a Monte Carlo simulation that has as input the observed proton energy spectrum (see Cummings et al. 2021 for more details). The dotted line is a fit to the data from 2007 forward that is used to estimate an uncertainty on each data point. The factor 1.193 shown in the equation in the figure accounts for the $120^{\circ}$ opening angle of the telescope and the minus sign corrects to depict the direction of flow. The additional $17 \mathrm{~km} \mathrm{~s}^{-1}$ corrects to the Sun's frame of reference. (e) Resulting CRS $V_{\mathrm{R}}$ for each roll. Also shown are the LECP 26 day averages of $V_{\mathrm{R}}$ from Krimigis et al. (2011). (f) Yearly weighted averages of the CRS (blue) and LECP (red) $V_{\mathrm{R}}$ values shown in panel (e).

magnetic flux at V2 does not change from the solar wind out to 6 au beyond the TS in the HSH, but the V1 values of magnetic flux decrease across the HSH. Burlaga et al. (2021) show that the magnetic flux observed at 1 au and that derived from PLS and MAG data at V2 in the HSH track remarkably well when the propagation time is accounted for.

The top panel of Figure 1 compares the V2 PLS and CG $V_{\mathrm{R}}$ speeds in the HSH. The blue trace shows LECP 5 day averages of $V_{\mathrm{R}}$ and the red diamonds show CRS $V_{\mathrm{R}}$ derived at each roll (Cummings et al. 2021). The black trace shows 5 day running averages of PLS 1 day average $V_{\mathrm{R}}$ values. Many differences between the V2 PLS and CG $V_{\mathrm{R}}$ values are apparent. The PLS values decrease slowly across the HSH and do not drop below $80 \mathrm{~km} \mathrm{~s}^{-1}$ until just before the HP. From 2009-2010 the CG $V_{\mathrm{R}}$ values increase to about $240 \mathrm{~km} \mathrm{~s}^{-1}$, twice the PLS $V_{\mathrm{R}}$ values. This difference was thought to be due to LECP ions being contaminated by $\mathrm{O}^{+}$(Richardson et al. 2013), but since the CRS and LECP CG $V_{\mathrm{R}}$ speeds are similar, $\mathrm{O}^{+}$contamination does not seem to be the cause. From 2011 to 2016 the average PLS and CG $V_{\mathrm{R}}$ speeds are similar, but the CG values show much more variation, with $V_{\mathrm{R}}$ changes of up to $200 \mathrm{~km} \mathrm{~s}^{-1}$ occurring within a few months. At 2016 the CG speeds drop systematically below the PLS speeds and average about half the PLS $V_{\mathrm{R}}$ from 2016 to the HP.

The bottom two panels of Figure 1 compare the magnetic flux observed at 1 au derived from the SPDF OMNI data set to the magnetic flux calculated from the Voyager data using the PLS (middle panel) and CG (bottom panel) $V_{\mathrm{R}}$ values. The 


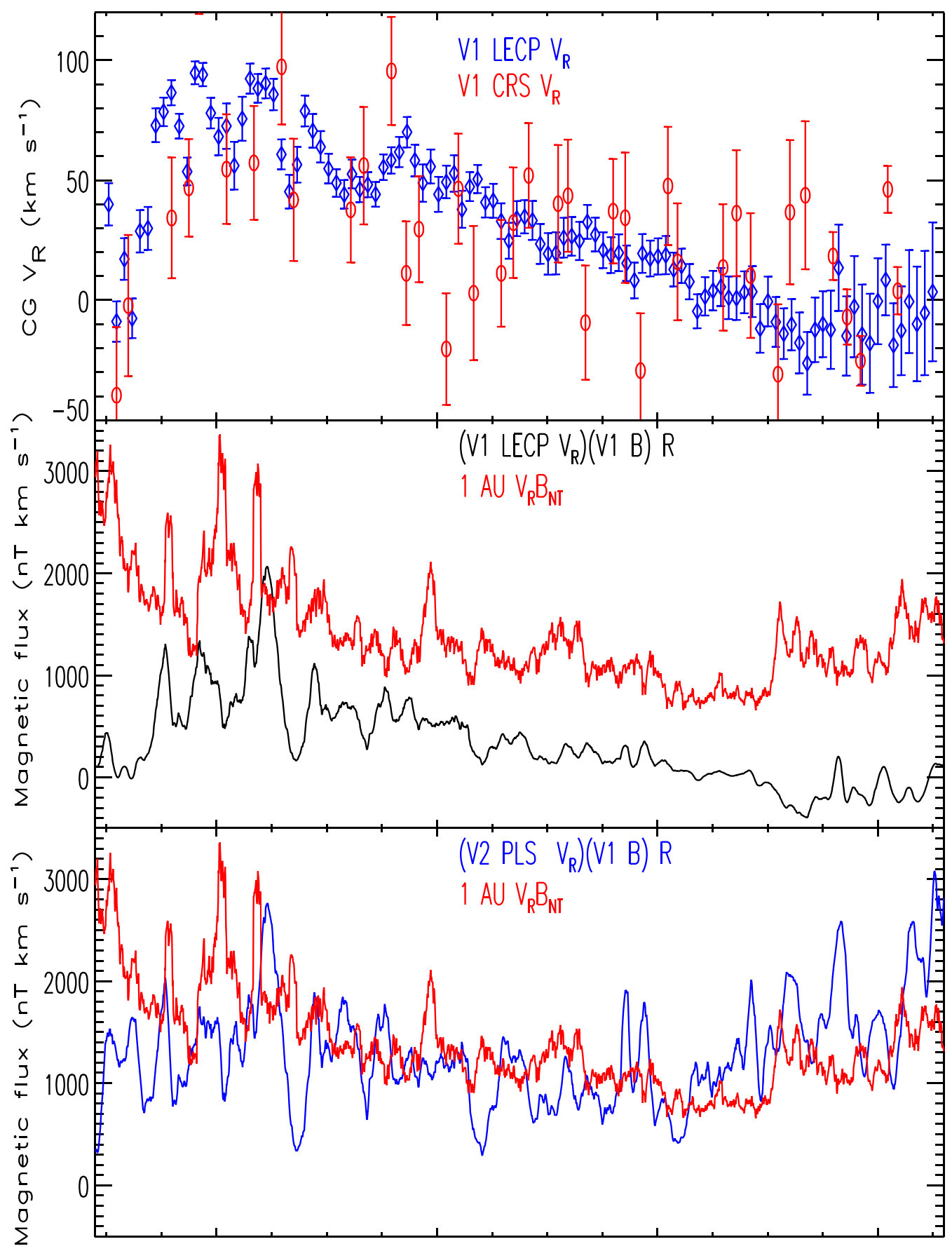

Figure 3. Top: V1 LECP CG 26 day average $V_{\mathrm{R}}$ (blue diamonds) and CRS CG $V_{\mathrm{R}}$ (red circles) from roll data, both with $1 \sigma$ error bars. Middle: 99 day running average of magnetic flux from 1 au (red) compared with 25 day running averages of the V1 magnetic flux calculated using the LECP speeds (black). The 1 au data are timeshifted forward $1 \mathrm{yr}$ to account for the propagation time. Bottom: 99 day running averages of magnetic flux from 1 au (red) compared with 25 day running averages of the V1 magnetic flux calculated using the V2 PLS speed profile and V1 magnetic field (blue). The 1 au data are time-shifted forward 1 yr to account for the propagation time.

panels show 99 day running averages of the 1 au magnetic flux $\mathrm{V}_{\mathrm{R}}\left|B_{\mathrm{NT}}\right|, 25$ day averages of the V2 magnetic flux $V|B| R$ using the PLS $V_{\mathrm{R}}$ values, and 25 day running averages of the V2 magnetic flux $V_{\mathrm{R}}|B| R$ using the LECP CG $V_{\mathrm{R}}$ values, where $B_{\mathrm{NT}}=$ sqrt $\left(B_{\mathrm{T}}^{2}+B_{\mathrm{N}}{ }^{2}\right)$ and $V_{\perp}=\operatorname{sqrt}\left(\mathrm{V}_{\mathrm{R}}^{2}+\mathrm{V}_{\mathrm{N}}{ }^{2}\right)$. Since $B$ is mainly in the $T$ direction, $V_{\mathrm{T}}$ does not contribute to magnetic flux transport; the Voyager $B_{\mathrm{R}}$ values are consistent with 0 over $95 \%$ of the time given the uncertainty in $B_{\mathrm{R}}$ of $0.06 \mathrm{nT}$ (Burlaga et al. 2021). $V_{\mathrm{N}}$ cannot be determined at $\mathrm{V} 2$ by LECP because the scan platform is in the RT plane, but since
$V_{\mathrm{R}}>V_{\mathrm{N}}$ by about a factor of 2 in the PLS data, this difference is assumed to be unimportant. We use 99 day running averages of the 1 au data to mimic the observed smoothing of solar wind variations with distance (Elliott et al. 2019).

The correlation in the middle panel between the 1 au and V2 magnetic flux profiles, although predicted by magnetic flux conservation, is remarkable given the large radial separation of the observations and suggests that little flux is removed by reconnection (Burlaga et al. 2021). The bottom panel compares the $1 \mathrm{au}$ magnetic flux to that calculated using the CG $V_{\mathrm{R}}$ 


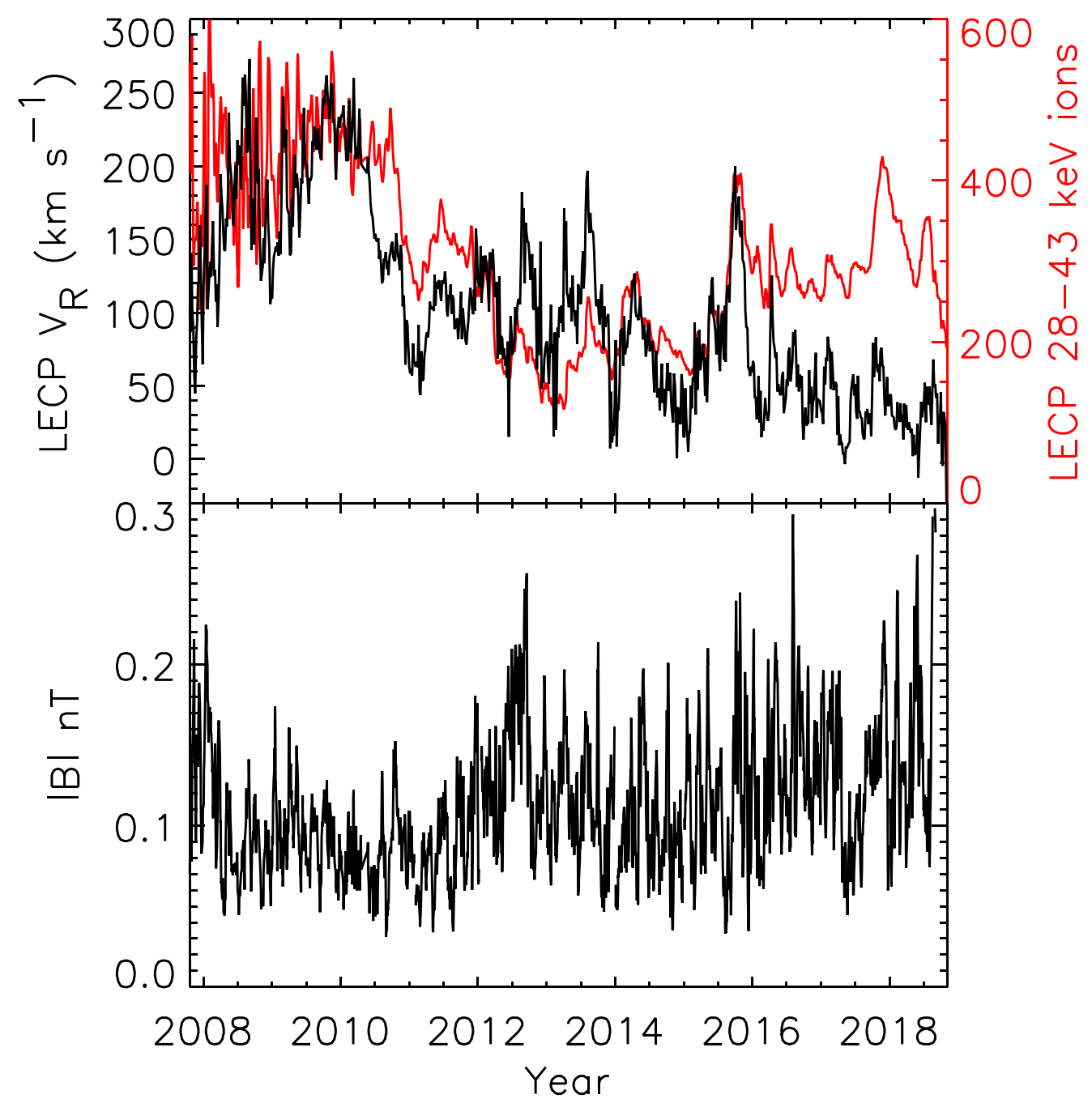

Figure 4. Top: V2 CG $V_{\mathrm{R}}$ at 5 day time resolution derived from $28-43 \mathrm{keV}$ LECP ion intensities (black) compared with 5 day running average $28-43 \mathrm{keV}$ LECP ion intensities (red). Bottom: 5 day running average of the V2 magnetic field magnitude.

values. The agreement is not as good, especially in the regions where PLS and LECP disagree. The large spike from 2009-2010 is not seen in the 1 au data, and neither is the decrease in magnetic flux after 2016 driven by the smaller values of CG $V_{\mathrm{R}}$. The large peaks and valleys in magnetic flux driven by the large $C G V_{R}$ changes are also not observed in the 1 au data. We conclude that the V2 PLS $V_{\mathrm{R}}$ values are more consistent with magnetic flux conservation than the $C G$ values.

Figure 2 compares V1 radial speeds from a new CG analysis of CRS data acquired during S/C rolls with V1 CG speeds from LECP. The CRS analysis is essentially identical to that carried out for V2 and described in detail in Cummings et al. (2021). The panels show the key parameters in the derivation. The counting rate of particles triggering the first detector of the LET A stack of detectors (and not triggering the fourth detector) is shown. Not depicted is similar data from LET D, the other telescope used in the analysis. An important parameter is $k_{D}$, a normalization factor for the LET $D$ rate that makes it as if LET A were occupying the position of LET D. The two telescopes have boresights that are $90^{\circ}$ apart and, importantly, have R-components with opposite signs. The Compton-Getting factor, $F$, is calculated from the measured spectrum for each roll day. The CG-derived $V_{\mathrm{R}}$ values agree reasonably well with those from LECP, as was the case for V2.
Figure 3 shows a similar comparison for V1 to that shown for V2 in Figure 1. The speeds available are 26 day average LECP CG speeds and the CRS speeds; as stated above the V1 PLS is not working. The top panel shows these speeds across the HSH. The speeds are lower than at V2 throughout the HSH, always $<100 \mathrm{~km} \mathrm{~s}^{-1}$, and the stagnation region with $V_{\mathrm{R}} \sim 0$ starts in 2010. The second panel compares 99 day running averages of the daily OMNI magnetic flux (roughly four solar rotations to smooth transient variations) with 25 day averages calculated with the V1 data. The V1 magnetic flux is well below that observed at 1 au almost everywhere in the $\mathrm{HSH}$. This difference in magnetic flux at 1 au and $\mathrm{V} 1$ persists for $7 \mathrm{yr}$, much longer than the roughly $2 \mathrm{yr}$ transit time of plasma from the Sun to the HP, so it seems that this difference cannot be a time-dependent effect. We ask the same question posed by Cummings et al. (2021): what if the CG $V_{\mathrm{R}}$ values are incorrect and there is no stagnation region? Although V2 crossed the HSH at a different heliolatitude than V1, was further back in the flank, and was at a different time in the solar cycle, we conjecture what the magnetic flux at V1 would look like if the $V_{\mathrm{R}}$ profile were the same as that observed by PLS at V2. We smoothed the V2 PLS speed profile and compressed it to fit the shorter V1 HSH transit time. The bottom panel of Figure 2 shows that the 1 au and magnetic flux values using the $\mathrm{V} 2 V_{\mathrm{R}}$ 
profile agree much better than when the $\mathrm{V} 1 \mathrm{CG} V_{\mathrm{R}}$ values are used.

\section{Discussion and Summary}

The magnetic flux conservation plots in Figures 1 and 3 show that the PLS $V_{\mathrm{R}}$ profile combined with the magnetic field observations match the 1 au observations well whereas the $\mathrm{CG}$ $V_{\mathrm{R}}$ values do not. In particular, the LECP magnetic flux values are low compared to the 1 au values everywhere at V1 and after $\sim 2014$ at V2. If the CG values were correct, this result would imply a large loss of magnetic flux due to reconnection. Although reconnection has been suggested to be important in the sector region of the $\mathrm{HSH}$, where heliospheric current sheet crossings are observed (Drake et al. 2017), no evidence for significant reconnection is observed in the MAG data (Burlaga et al. 2021). The average magnetic field remains constant across both the V1 and V2 HSH crossings, consistent with a slowly changing $V_{\mathrm{R}}$. Cummings et al. (2021) point out that the CG $V_{\mathrm{R}}$ values are correlated with the CRS $>0.5 \mathrm{MeV}$ ion intensity. Figure 4 shows that this correlation also holds for the low energy 28-43 keV LECP ions; the top panel shows LECP $V_{\mathrm{R}}$ (black) and particle intensity (red). These variations in particle intensity and $V_{\mathrm{R}}$ do not correlate well with changes in $\mathrm{B}$ (bottom panel), except for the large ICME observed near the end of 2015. After 2012, the HSH is dominated by solar wind dynamic pressure pulses that are very well correlated with changes in the $\mathrm{keV}$ and $\mathrm{MeV}$ particle intensities but not with $\mathrm{B}$ (Richardson et al. 2018). These pressure pulses correspond to higher $\mathrm{CG} V_{\mathrm{R}}$ regions as shown in Figure 4, but only a few (such as the 2015 ICME) show increases in PLS $V_{\mathrm{R}}$ and $B$. After 2016, $V_{\mathrm{R}}$ decreases even though the particle intensity increases to a plateau before the HP. A puzzle is whether something about these pressure and intensity increases could affect particle anisotropies and thus the $\mathrm{CG}$ speeds.

In summary, the PLS and CG V2 $V_{\mathrm{R}}$ values differ significantly, but only the PLS values are consistent with magnetic flux conservation. The PLS $V_{\mathrm{R}}$ profile decreases slowly and monotonically from the TS to the HP. CG $V_{\mathrm{R}}$ values from LECP and CRS, derived from different energy particles, are similar. They show large speed variations over time periods of several months, are over $200 \mathrm{~km} \mathrm{~s}^{-1}$ for a $1 \mathrm{yr}$ period in 2009 , and the CG $V_{\mathrm{R}}$ values are lower than those from PLS for the $2 \mathrm{yr}$ period before the HP. We show that V1 LECP and CRS speeds also agree reasonably well. The $V_{\mathrm{R}}$ values from
PLS are consistent with magnetic flux conservation, matching $1 \mathrm{au}$ values very well, whereas those from the CG are not. We do not understand why the $\mathrm{CG}$ values are consistent at different particle energies, and yet are still apparently unreliable, and look forward to future studies of this issue.

J.D.R. was supported under NASA contract 959203 from the Jet Propulsion Laboratory to the Massachusetts Institute of Technology and by grants 2019-064 (UAH) and SUB000318 (IMAP). J.D.R., M.O., and A.C.C. acknowledge support from NASA grant 18-DRIVE18_2-0029, Our Heliospheric Shield, 80NSSC20K0603. A.C.C. and E.C.S. were supported under NASA award number NNN12AA01C. L.F.B. is supported by NASA contract \#80GSFC19C0012.

\section{ORCID iDs}

J. D. Richardson (iD https://orcid.org/0000-0003-4041-7540

A. C. Cummings (ib https://orcid.org/0000-0002-3840-7696

L. F. Burlaga (D) https://orcid.org/0000-0002-5569-1553

J. Giacalone (i) https://orcid.org/0000-0002-0850-4233

M. Opher (i) https://orcid.org/0000-0002-8767-8273

E. C. Stone (ib https://orcid.org/0000-0002-2010-5462

\section{References}

Bridge, H. S., Belcher, J. W., Butler, R. J., et al. 1977, SSRv, 21, 259 Burlaga, L. F., Berdichevsky, D. B., Jian, L. K., et al. 2021, ApJ, 906, 119 Bzowski, M., Swaczyna, P., Kubiak, M. A., et al. 2015, ApJS, 22, 28 Cummings, A. C., Stone, E. C., Richardson, J. D., et al. 2021, ApJ, 906, 126 Decker, R. B., Krimigis, S. M., Roelof, E. C., \& Hill, M. E. 2012, Natur, 489, 124

Drake, J. F., Swisdak, M., Opher, M., \& Richardson, J. D. 2017, ApJ, 837, 159 Elliott, H. A., McComas, D. J., Zirnstein, E. J., et al. 2019, ApJ, 885, 156 Fuselier, S. A., Galli, A., Richardson, J. D., et al. 2021, ApJL, 915, L26

Krimigis, S. M., Decker, R. B., Roelof, E. C., et al. 2019, NatAs, 3, 997

Krimigis, S. M., Roelof, E. C., Decker, R. B., \& Hill, M. E. 2011, Natur, 474, 359

Parker, E. N. 1963, Interplanetary Dynamical Process (New York: Wiley)

Richardson, J. D., Belcher, J. W., Burlaga, L. F., et al. 2020, JPhCS, 1620, 012016

Richardson, J. D., Belcher, J. W., Cummings, A. C., Decker, R., \& Stone, E. C. 2018, JPhCS, 1100, 012019

Richardson, J. D., Belcher, J. W., Garcia-Galindo, P., \& Burlaga, L. F. 2019, NatAs, 3, 1019

Richardson, J. D., Burlaga, L. F., Decker, R. B., et al. 2013, ApJL, 762, L14 Richardson, J. D., \& Decker, R. B. 2014, ApJ, 792, 126

Stone, E. C., \& Cummings, A. C. 2011, ICRC (Beijing), 12, 29

Stone, E. C., Vogt, R. E., McDonald, F. B., et al. 1977, SSRv, 21, 355 